\title{
可穿戴气液两用甲醇智能电化学传感器的制备与应用研究
}

朱楠 ${ }^{1}$, 马鸿婷 1 , 陶欢诺 ${ }^{1}$, 王雪 2 , 宿艳 ${ }^{2, *}$

1 大连理工大学张大暲学院, 辽宁大连 116024

2 大连理工大学化工学院, 辽宁大连 116024

摘要: 甲醇作为一种基础化工原料, 是生产所需的常用溶剂, 也是醇类燃料电池的常用燃料之一。然而其具有挥发 性, 会对人体的神经系统产生毒化作用, 因此, 实时监测环境中的甲醇蒸气浓度具有重要意义。本文在科研成果的 基础上创新设计了一个综合实验——可穿戴智能气液双功能甲醇电化学传感器。实验过程包括无机材料合成、丝网 印刷制备、仪器分析表征、电化学传感检测等内容, 涵盖多门化学课程主要相关知识点, 具有很好的综合性和学科 前沿性。经过此实验训练, 学生不仅能巩固相关化学基础知识, 掌握石墨烯材料的合成与表征技能, 还能了解电化 学传感器的工作原理, 激发学生对科学研究的兴趣。

关键词: 可穿戴传感器; 甲醇; 电化学; 气液两用

中图分类号: G64; O6

\section{Preparation and Application of Wearable Smart-Sensors for Vaporl Liquid Amphibious Methanol Monitoring}

\author{
Nan Zhu ${ }^{1}$, Hongting Ma ${ }^{1}$, Huannuo Tao ${ }^{1}$, Xue Wang ${ }^{2}$, Yan Su ${ }^{2,}{ }^{*}$ \\ ${ }^{1}$ Zhang Dayu School of Chemistry, Dalian University of Technology, Dalian 116024, Liaoning Province, China. \\ ${ }^{2}$ School of Chemical Engineering, Dalian University of Technology, Dalian 116024, Liaoning Province, China.
}

\begin{abstract}
Methanol, a basic chemical raw material, is a common solvent in production and one of the common fuels of alcohol fuel cells. However, it is volatile and can cause toxicity to the human nervous system. Therefore, real-time monitoring of the concentration of methanol vapor in the environment is of great significance. In this paper, a comprehensive experiment "a wearable intelligent high selectivity gas-liquid bifunctional methanol electrochemical sensor" was designed on the basis of scientific research achievements. The experiment includes inorganic material synthesis, screen printing preparation, instrumental analysis and characterization, electrochemical sensing and detection, covering the main related knowledge points of several chemistry courses, thus is very comprehensive and cutting-edge. Through this experiment, students can not only consolidate the basic knowledge of chemistry, master the synthesis and characterization skills of graphene materials, but also understand the working principle of electrochemical sensors, which greatly stimulate students' interests in scientific research.
\end{abstract}

Key Words: Wearable sensors; Methanol; Electrochemistry; Vapor/liquid

由于课程设置等原因, 在无机化学实验中, 学生们合成过沸石分子篮等材料, 但缺乏对材料的 进一步结构表征和性能测试; 在分析化学实验中, 学生们应用了电位分析法检测水中微量氟离

收稿: 2021-10-25; 录用: 2021-11-09; 网络发表: 2021-12-17

”通讯作者, Email: susu@dlut.edu.cn

基金资助：大连理工大学研究生教改基金项目(JG_2019095); 大连市科技创新基金(2019J12SN54); 国家级质量工程建设项目(ZL2021346) 
子, 但缺乏对循环伏安法等其他电化学分析方法的实践应用; 同时, 无机分析实验多为传统的经典 实验, 缺乏对学科前沿的紧密追踪和实际应用 ${ }^{[1-3]}$ 。近年来, 新型可穿戴电子设备作为学科前沿探究 热点, 有效地将无机合成方法与传统的电化学分析方法相结合, 集高灵敏、舒适便携于一体, 能够 实时分析检测佩戴者周围环境中的有毒有害气体。

甲醇在工业生产和日常生活中应用广泛，然而，作为常见的有一定生理毒性的有机挥发性化合 物(VOC), 吸入或皮肤吸收大量甲醇可能导致人失明、头痛、甚至死亡 ${ }^{[4]}$ 。甲醇的常规检测方法有高 效液相色谱法、气相色谱法、紫外-可见分光光度法等。然而, 这些检测方法存在仪器设备笨重、无 法实现实时在线监测, 以及小型化困难等问题 ${ }^{[5,6]}$ 。

基于甲醇电化学反应的传感器应运而生 ${ }^{[7-9]}$, 文献表明, 金属铂(Pt) 以其独特的电催化性能已成 为甲醇电化学氧化反应的理想催化剂之一[10]。但是, 纯Pt催化剂价格昂贵, 而Pt纳米粒子易被含碳 中间产物 $(\mathrm{CO})$ 吸附而 “中毒”, 使其催化性能大大下降 ${ }^{11]}$, 极大地限制了传感器的实际应用。为解 决这一弊端, 有必要对Pt催化剂予以修饰。如选择合适的导电基底, 将Pt催化剂生长和针定在导电 基底上, 提高电催化剂的表面积, 进而提高甲醇氧化的电流效率。在炭黑、碳纳米管、还原氧化石 墨烯(rGO) ${ }^{[12]}$ 等各种碳二维纳米材料中, 还原氧化石墨烯因其自身优异的理化性质, 如高的体积 比、优异的电荷载流子迁移率、结构柔韧性和超薄性脱颖而出, 成为负载铂纳米颗粒的理想载 体。已有文献报道将石墨烯作为导电基底, 用于液体中甲醇浓度的检测。例如, 研究者制备了负载 $\mathrm{Pt}$ 纳米粒子的石墨烯材料 ${ }^{[13]}$, 在实验过程中, 先将氧化石墨烯 $(\mathrm{GO})$ 还原, 再将Pt纳米粒子均匀分散 在其表面, 所制备的催化剂对甲醇氧化显示出更高的电催化活性。但该传感器仅用于水相中甲醇的 检测, 且不具备可穿戴的灵活性和实际应用性。基于此, 本文提出了可穿戴式电化学甲醇传感器的 设计思路, 即通过丝网印刷技术在基底上打印制备电极, 并在工作电极表面修饰负载铂纳米粒子的 石墨烯 $(\mathrm{Pt} / \mathrm{rGO})$, 得到很好的甲醇催化性能, 也可以进一步打印到手套上, 将信号传输至手机等移动 设备, 真正实现实时监测的目的。

本工作结合了基础性的理论知识研究和前沿性的化学器件制作。在实验内容上, 展现了从无机 材料合成、仪器分析表征 ${ }^{[14]}$ 、电化学性能测试到传感实践应用的巧妙构思, 涵盖多门化学课程主要 相关知识点, 有效培养学生综合运用化学专业知识解决实际问题的能力。在实验进程上, 展现出简 单、易重复的鲜明特色, 这两点对于本科综合实验课程来讲难能可贵, 方便为其他本科院校开设综 合实验课程提供思路参考。此外, 结合科技前沿的化学器件制作能大大激发学生对科学研究的兴 趣, 培养学生发现问题、分析问题和解决问题的能力。

\section{1 实验部分}

\section{1 实验原理}

甲醇氧化的机理过程主要以下面的电化学反应为基础:

$$
\begin{aligned}
& \mathrm{CH}_{3} \mathrm{OH}+\mathrm{H}_{2} \mathrm{O} \stackrel{\mathrm{Pt}}{\rightarrow} \mathrm{CO}_{2}+6 \mathrm{H}^{+}+6 \mathrm{e}^{-} \\
& \mathrm{CH}_{3} \mathrm{OH}_{\text {solution }}+2 \mathrm{Pt} \rightarrow \mathrm{Pt}-\left(\mathrm{CH}_{2} \mathrm{OH}\right)_{\text {ads }}+\mathrm{Pt}-\mathrm{H} \\
& \mathrm{Pt}-\left(\mathrm{CH}_{2} \mathrm{OH}\right)_{\mathrm{ads}}+2 \mathrm{Pt} \rightarrow \mathrm{Pt}_{2}-(\mathrm{CHOH})_{\mathrm{ads}}+\mathrm{Pt}-\mathrm{H} \\
& \mathrm{Pt}-(\mathrm{CHOH})_{\mathrm{ads}}+2 \mathrm{Pt} \rightarrow \mathrm{Pt}_{3}-(\mathrm{COH})_{\text {ads }}+\mathrm{Pt}-\mathrm{H} \\
& \mathrm{Pt}_{3}-(\mathrm{COH})_{\mathrm{ads}} \rightarrow\left\{\begin{array}{l}
\mathrm{Pt}_{2}-(\mathrm{CO})_{\mathrm{ads}}+\mathrm{Pt}+\mathrm{H}^{+}+\mathrm{e}^{-} \\
\mathrm{Pt}-(\mathrm{CO})_{\mathrm{ads}}+2 \mathrm{Pt}+\mathrm{H}^{+}+\mathrm{e}^{-}
\end{array}\right. \\
& \mathrm{Pt}+\mathrm{H}_{2} \mathrm{O} \rightarrow \mathrm{Pt}-(\mathrm{OH})_{\mathrm{ads}}+\mathrm{H}^{+}+\mathrm{e}^{-} \\
& \mathrm{Pt}-(\mathrm{CO})_{\mathrm{ads}}+\mathrm{Pt}-(\mathrm{OH})_{\mathrm{ads}} \rightarrow 2 \mathrm{Pt}+\mathrm{CO}_{2}+\mathrm{H}^{+}+\mathrm{e}^{-}
\end{aligned}
$$

由式1可见, 甲醇氧化过程是一个由催化剂驱动的六电子转移电化学反应。首先, 甲醇被Pt催化 分解, 导致 $\mathrm{C}-\mathrm{H}$ 键断裂。在电催化过程中 (式2-4) ${ }^{[15]}$, 将会发生一系列的平行反应, 并形成各种氧 
化中间产物。之后, 所吸附的中间产物再分别经过 $\mathrm{CO}$ 路径(式5)或非 $\mathrm{CO}$ 路径(式6)进一步氧化成 $\mathrm{CO}_{2}{ }^{[16]}$ 。对于传统的纯Pt催化剂, 中间氧化产物(CO)会在催化剂表面聚集, 导致催化剂中毒, 使得 催化剂的电催化活性变差。因此, 将Pt纳米粒子负载在还原氧化石墨烯基体上, 由于石墨烯材料的 优良导电性和 $\mathrm{P}$ 纳米粒子的优良分散性, 可减轻氧化中间产物的聚集, 改善CO中毒现象, 保持催化 剂良好的电催化活性。

本实验设计提出了可穿戴式电化学甲醇传感器的设计思路(图1), 通过丝网印刷技术在基底上打 印制备电极, 并在工作电极表面修饰负载 $\mathrm{Pt}$ 纳米粒子的石墨烯基材料 $(\mathrm{Pt} / \mathrm{rGO})$, 实现了该传感系统对 于甲醇的催化、传感性能, 其可以进一步打印到手套上, 而后再经过弯曲等实际测试其实用性; 也 可将信号传输至手机等移动设备, 真正实现实时监测环境中甲醇的目的。
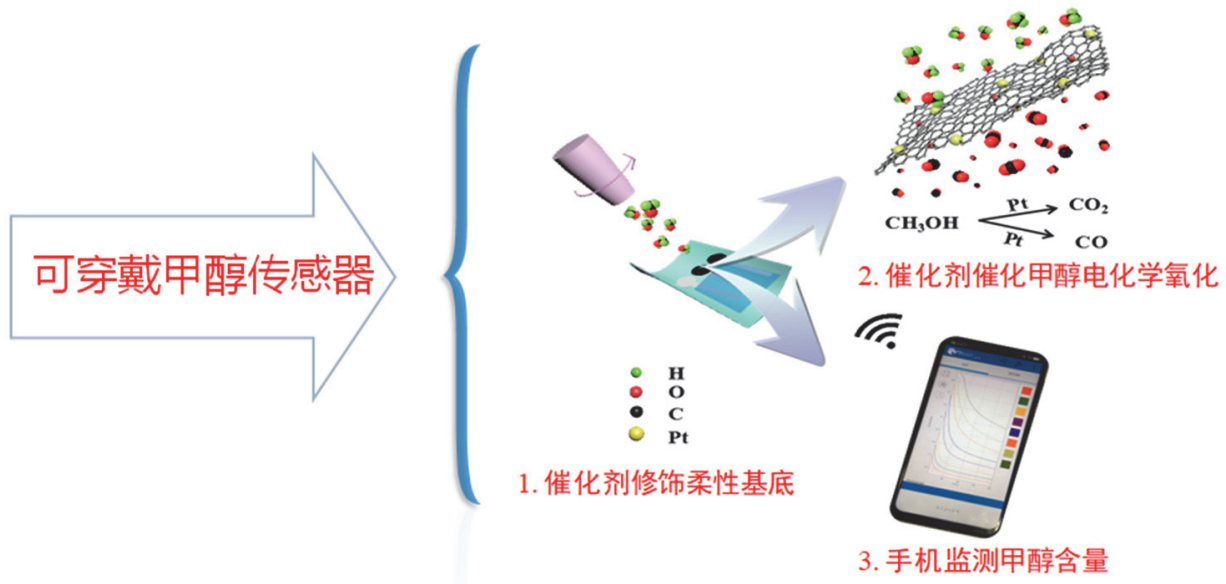

图1 可穿戴智能高选择性气液两相甲醇电化学传感器工作示意图

\section{2 药品与材料}

药品: 过氧化氢 $\left(\mathrm{H}_{2} \mathrm{O}_{2}\right.$, 质量分数 $\left.\omega=30 \%\right)$, 浓硫酸 $\left(\mathrm{H}_{2} \mathrm{SO}_{4}\right.$, 质量分数 $\left.\omega=98 \%\right)$, 盐酸 $(\mathrm{HCl}$, 质 量分数 $\omega=0.1 \%)$, 硝酸 $\left(\mathrm{HNO}_{3}\right.$, 分析纯), 高锰酸钾 $\left(\mathrm{KMnO}_{4}, \mathrm{Aesar}\right.$, 分析纯), 甲醇(分析纯), 过硫 酸钾 $\left(\mathrm{K}_{2} \mathrm{~S}_{2} \mathrm{O}_{4}\right.$, 分析纯), 五氧化磷 $\left(\mathrm{P}_{2} \mathrm{O}_{5}\right.$, 分析纯), 氮气 $\left(\mathrm{N}_{2}\right)$, 氢氧化钠溶液(分析纯), 六水合氯铂 酸 $\left(\mathrm{H}_{2} \mathrm{PtCl}_{4} \cdot 6 \mathrm{H}_{2} \mathrm{O}\right.$, Sigma Aldrich, 分析纯), 全氟磺酸聚四氟乙烯Nafion (Sigma Aldrich), 碳素墨 水, 银/氯化银油墨(BY-2000H, 上海宝银), 天然石墨粉。

材料: 聚对苯二甲酸乙二酯塑料(PET), 导电银胶, 导线, 尼龙手套, 绝缘胶带。

\section{3 仪器和表征方法}

\subsection{1 仪器}

半自动丝网印刷机(4060垂直丝网印刷机), 气体流量计, 超声波清洗仪(洁盟), 上海辰华电化学 工作站(CHI 1242C), X射线衍射仪(XRD, rigakud/MAX-2400), 紫外-可见分光光度计(UV8453), 扫 描电子显微镜(SEM, Nova Nanosem 450)。

\subsection{2 表征方法}

XRD分析：采用粉末 X射线衍射仪(XRD)对催化剂组成和晶体结构进行表征;

紫外光谱分析：采用紫外-可见分光光度法(UV-Vis)确认氧化石墨烯是否被还原为还原氧化石 墨烯;

形貌分析：采用扫描电子显微镜(SEM)研究催化剂样品的形貌;

在实验学时和仪器设备满足的条件下, 可完成全部表征分析; 在不方便进行电镜等仪器测试 时, 可通过完成XRD和UV-Vis实现对催化剂的初步表征。 


\section{4 实验步骤}

\subsection{1 丝网印刷电极的制备}

传感器电极由商用半自动丝网印刷机(4060垂直丝网印刷机)制备。首先, 通过蚀刻模板刷墨(厚 度 $1 \mathrm{~mm}$ ), 将 $\mathrm{Ag} / \mathrm{AgCl}$ 油墨印刷到基材(PET或乙腈手套)上, 确定参比电极和连接区域。其次, 采用 相同的过程印刷碳素油墨(电极印刷面积 $5 \mathrm{~mm}^{2} \times 12$ ), 并将其作为工作电极和对电极。最后, 印刷绝 缘层的第三层, 其厚度约为 $50 \mu \mathrm{m}$, 由网印版与刮板刀之间的距离决定。每次丝网印刷后, 将电极置 于 $80^{\circ} \mathrm{C}$ 下固化 $30 \mathrm{~min}$ 。

\section{4 .2 氧化石墨烯 $(\mathrm{GO})$ 和铂负载的还原氧化石墨烯(Pt/rGO)合成}

$\mathrm{GO}$ 由改进的Hummer方法制备 ${ }^{[17]}$ : 首先, 在 $80{ }^{\circ} \mathrm{C}$ 下将 $2.5 \mathrm{~g} \mathrm{~K}_{2} \mathrm{~S}_{2} \mathrm{O}_{4}$ 和 $2.5 \mathrm{~g} \mathrm{P}_{2} \mathrm{O}_{5}$ 放入 $7.5 \mathrm{~mL} \mathrm{H}_{2} \mathrm{SO}_{4}$ 中。再将 $5.0 \mathrm{~g}$ 天然石墨在连续摚拌 $3 \mathrm{~h}$ 的条件下添加到原溶液中。冷却至室温后, 用蒸馏水冲洗混合 物, 直到 $\mathrm{pH}$ 达到 7 。干燥过夜后再收集混合物。其次, 将上述 $1.0 \mathrm{~g}$ 混合物在冰水浴的条件下缓慢地 添加到 $23 \mathrm{~mL}$ 的硫酸中, 然后在连续摚拌下将 $3.0 \mathrm{~g}$ 高锰酸钾添加到混合物中。第三, 将混合物转移到 $250 \mathrm{~mL}$ 圆底烧瓶中, 并在 $35^{\circ} \mathrm{C}$ 搅拌 $2 \mathrm{~h}$ 。随着时间的推移, 混合物会变得更浓, 难以摚拌。反应结 束后加入 $180 \mathrm{~mL}$ 蒸馏水继续搅拌 $15 \mathrm{~min}$, 得到灰褐色混合物, 再加入 $2.5 \mathrm{~mL} 30 \%$ 的 $\mathrm{H}_{2} \mathrm{O}_{2}$ 。最后, 用 $250 \mathrm{~mL} 0.1 \%(w) \mathrm{HCl}$ 溶液洗涤悬浮液, 再通过离心得到黄色 $\mathrm{GO}$ 悬浮液。

$\mathrm{Pt} / \mathrm{rGO}$ 复合材料的制备如下 ${ }^{[18]}$ : 将 $105 \mathrm{mg}$ 的 $\mathrm{GO}$ 和 $143 \mathrm{mg} \mathrm{H}_{2} \mathrm{PtCl}_{4} \cdot 6 \mathrm{H}_{2} \mathrm{O}$ 添加到 $250 \mathrm{~mL}$ 蒸馏水 中。用氢氧化钠溶液调节混合物 $\mathrm{pH}$ 为 10 。然后, 添加 $0.84 \mathrm{~g} \mathrm{NaBH}_{4}$ 到混合物中作为还原剂, 并将溶 液在室温下摚拌 $2 \mathrm{~h}$, 再用蒸馏水清洗几次, 抽滤, 烘干。

实验过程中因涉及过氧化氢、高锰酸钾、浓硫酸等易制毒易制爆危险化学品, 要求学生务必查 阅化学品的安全技术说明书(MSDS), 在老师的监督下取用药品, 完成实验操作。

\subsection{3 铂负载的还原氧化石墨烯 $(\mathrm{Pt} / \mathrm{rGO})$ 电极的制备}

在使用之前, 将 $15 \mathrm{mg} \mathrm{Pt} / \mathrm{rGO}$ 催化剂在浴流混合器中与 $300 \mu \mathrm{L}$ 含有 $30 \mathrm{mg}$ Nafion的蒸馏水中混合 (超声 $10 \mathrm{~min}$ 亦可)。随后, 将 $2 \mu \mathrm{L}$ 的混合物滴涂在工作电极上三次, 每次在 $60{ }^{\circ} \mathrm{C}$ 下干燥 $5 \mathrm{~min}$ 。若用 来检测气体, 则还需用Nafion连续均匀覆盖在三个电极上并干燥。

\subsection{4 铂负载的还原氧化石墨烯 $(\mathrm{Pt} / \mathrm{rGO})$ 电极的电化学性能测试}

采用循环伏安法 $(\mathrm{CV})$ 和电流曲线 $(i-t)$ 法(在 CV正向扫描的峰值电位处测量)研究 $\mathrm{Pt} / \mathrm{rGO}$ 基复合材 料进行甲醇氧化的电化学行为。

\subsection{5 电化学甲醇传感器的实时检测}

液体测量装置: 甲醇液体试样可由无水甲醇与去离子水按一定比例混合配制。测量过程只需将 传感系统完全浸入样品中, 并在实验过程中持续摚拌即可。

气体测量装置: 甲醇蒸气试样可采用恒定的氮气流将其从甲醇水溶液中吹出(图2)。同时, 用气 体流量计控制氮气流量, 将吹出的甲醇蒸气用一个已安装有传感器的密封盒收集, 实时测量甲醇蒸 气的浓度(可使用气相色谱定量甲醇浓度)。

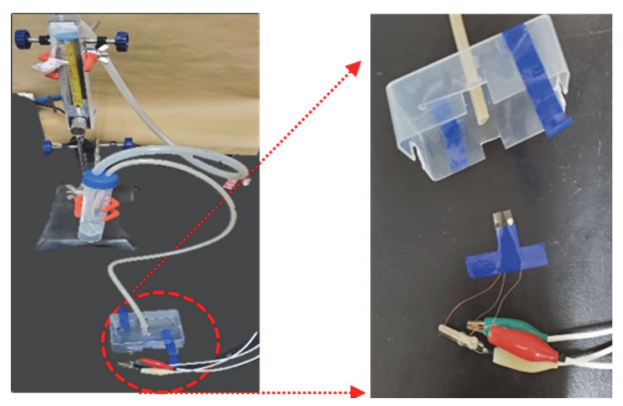

图2 气体测量装置图 


\section{2 结果与讨论}

\section{1 催化剂的分析表征}

选取电化学检测性能好的材料进行表征(图3)。SEM分析表明, Pt纳米粒子均匀地分散在石墨烯 表面, 粒径为8-15 $\mathrm{nm}$ (图3a)。XRD可用来分析表征催化剂的纳米晶型, 图3b显示在 $36.42^{\circ}$ 、 $46.31^{\circ} 、 67.74^{\circ} 、 81.59^{\circ}$ 和 $86.18^{\circ}$ 处出现尖峰, 分别对应于Pt的(111)、(200)、(220)、(311)和(222)晶 面。而且, 相比于未掺杂 $\mathrm{Pt}$ 催化剂, 所制备的 $\mathrm{Pt} / \mathrm{rGO}$ 催化剂在对应于 $\mathrm{GO}(001)$ 晶面的峰(11.72)消 失, 说明 $\mathrm{GO}$ 在制备过程中已被还原。图3c表明, 掺杂 $\mathrm{Pt}$ 的催化剂吸收峰红移, 其峰值由 $231 \mathrm{~nm}$ 红移 到 $268 \mathrm{~nm}$, 说明 $\mathrm{GO}$ 成功还原为 $\mathrm{rGO}$ 。
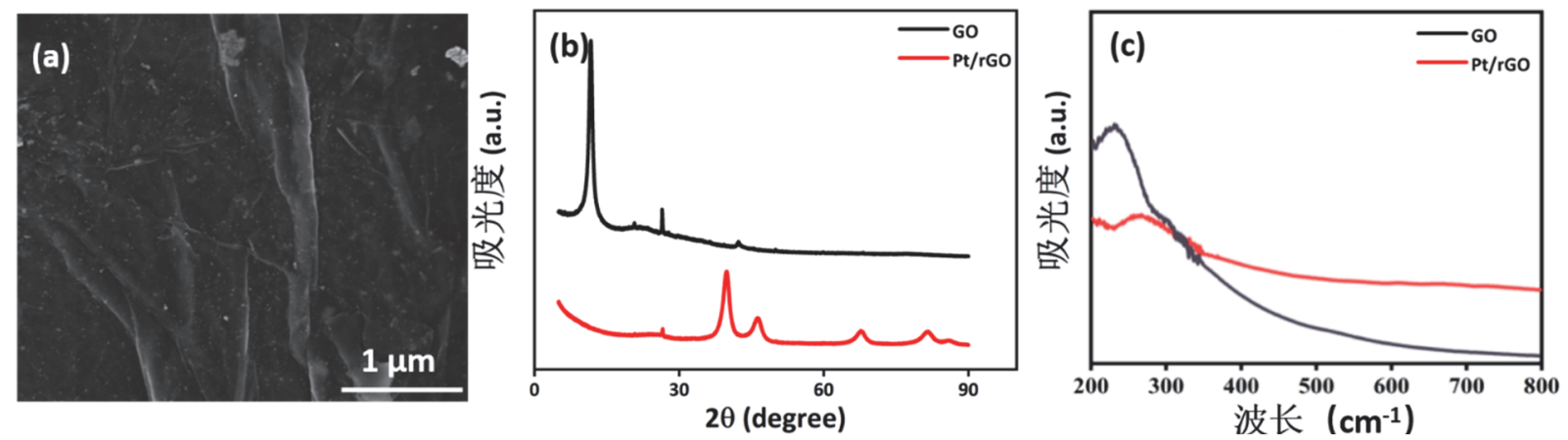

图3 Pt/rGO催化剂的表征

(a) SEM; (b) XRD; (c) UV-Vis

如果本科实验教学不具备使用SEM表征的条件, 可联合XRD与UV-Vis对所制备的Pt/rGO催化剂 进行表征分析。

\section{2 甲醇传感器的电化学性能}

以甲醇气体为例, 通过典型的电化学甲醇氧化循环伏安曲线的对比, 可以发现, 本工作所制备 的催化剂在正向扫描和负向扫描的过程中出现了两个明显的氧化峰(图4a), 表明该催化剂对于甲醇 的优异催化性能。其中, 正向扫描期间的阳极峰所对应的是甲醇被完全氧化成二氧化碳 $\left(\mathrm{CO}_{2}\right)$ 的 过程, 负向扫描期间的阳极峰则对应了去除不完全氧化物质, 如一氧化碳(CO)的过程。在正向阳极 峰电位下, 电流密度会随着甲醇浓度的升高而变大(图4b), 并在一定的甲醇浓度 (甲醇与水体积比 为 $1 \%-10 \%$ )范围内呈现出对应的线性关系(图4c)。

\section{3 可穿戴甲醇电化学传感器的稳定性和环境适应性}

采用丝网印刷的方法将三电极的传感系统打印在PET或柔性手套基底上, 将负载Pt纳米粒子的石 墨烯催化剂通过滴涂方式修饰在传感器工作电极表面, 用Nafion溶液将三个电极连接起来, 烘干后
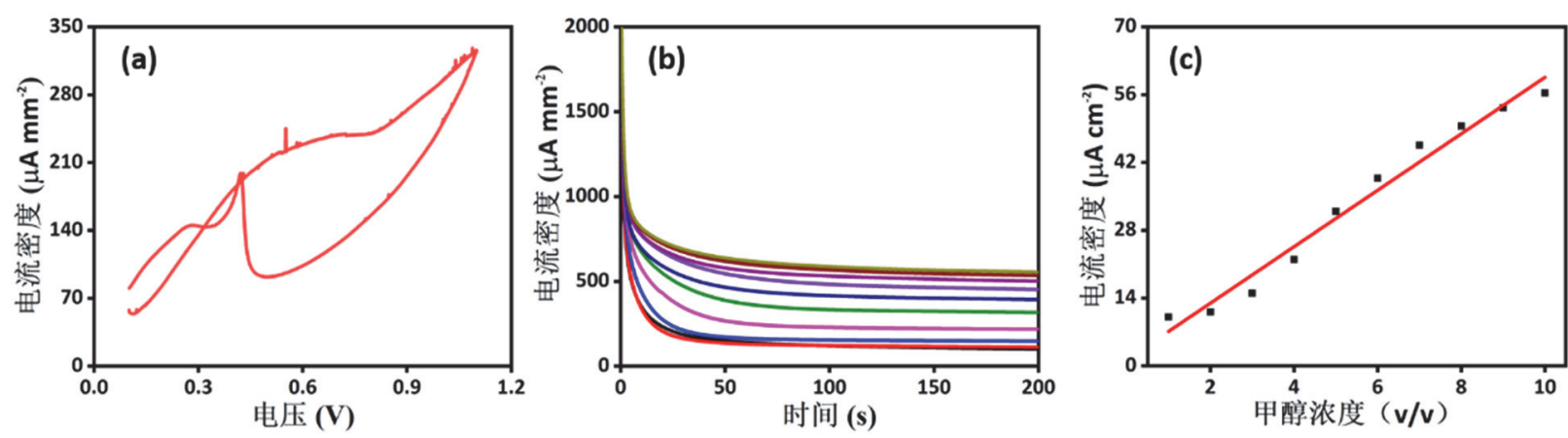

图4 Pt/rGO电极在甲醇环境下的电化学性能测试

(a) 循环伏安曲线(气体环境中甲醇浓度为 $5 \% v / v$ ); (b) 电流-时间曲线(气体环境中甲醇浓度为 $1 \%-10 \%$ ); (c) 电流密度与甲醇浓度的线性关系 
可获得可穿戴智能气液双功能甲醇电化学传感器。本传感器能够在长时间的测试中保持恒定的环境适 应性, 周围环境的温度(图5a)和湿度(图5b)变化对于传感性能的影响基本可以忽略, 这一结果极大地 保证了传感系统在日常生产和生活中的应用性，为该传感系统的大规模使用提供了理论基础。
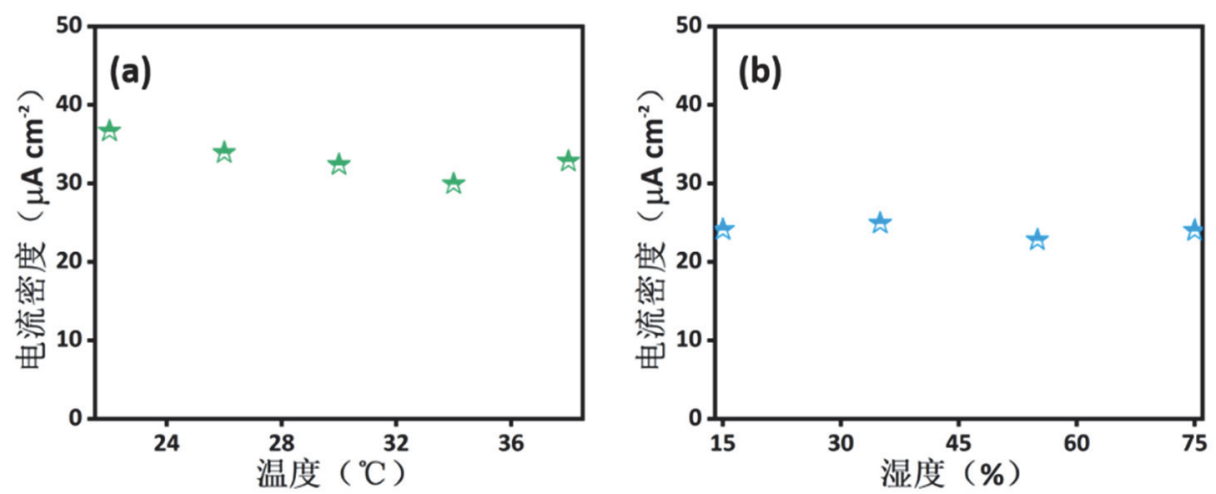

图5 可穿戴甲醇电化学传感器的稳定性实验

(a) 环境温度对稳定性影响测试; (b) 环境湿度对稳定性影响测试

\section{4 甲醇传感器的电化学性能}

将该可穿戴智能气液双功能甲醇电化学传感器与EmStatBlue电化学工作站相连后, 可通过蓝牙 装置, 将测试所得信号传输至移动手机上, 从而实现无线、实时测试(图6a)。传感性能与传统辰华电 化学工作站相比无差异, 该结果表明传感器脱离了大型电化学工作站的束缚, 可以在任意场合条件 下, 通过蓝牙装置, 用移动手机对周围环境中甲醇气体浓度进行监测, 真正做到了可穿戴实时监测 的目的, 为该传感器的实际应用提供了实验基础。同时由于导电油墨与手套基底的良好结合性, 传 感器在百余次弯曲过程中, 性能无明显衰减(图6b), 在50次 $10 \%$ 的拉伸测试中, 传感性能也保持良好 (图6c), 说明该传感器可以很好地适应日常生产生活的需要, 极大地提升了该传感器的实用性。
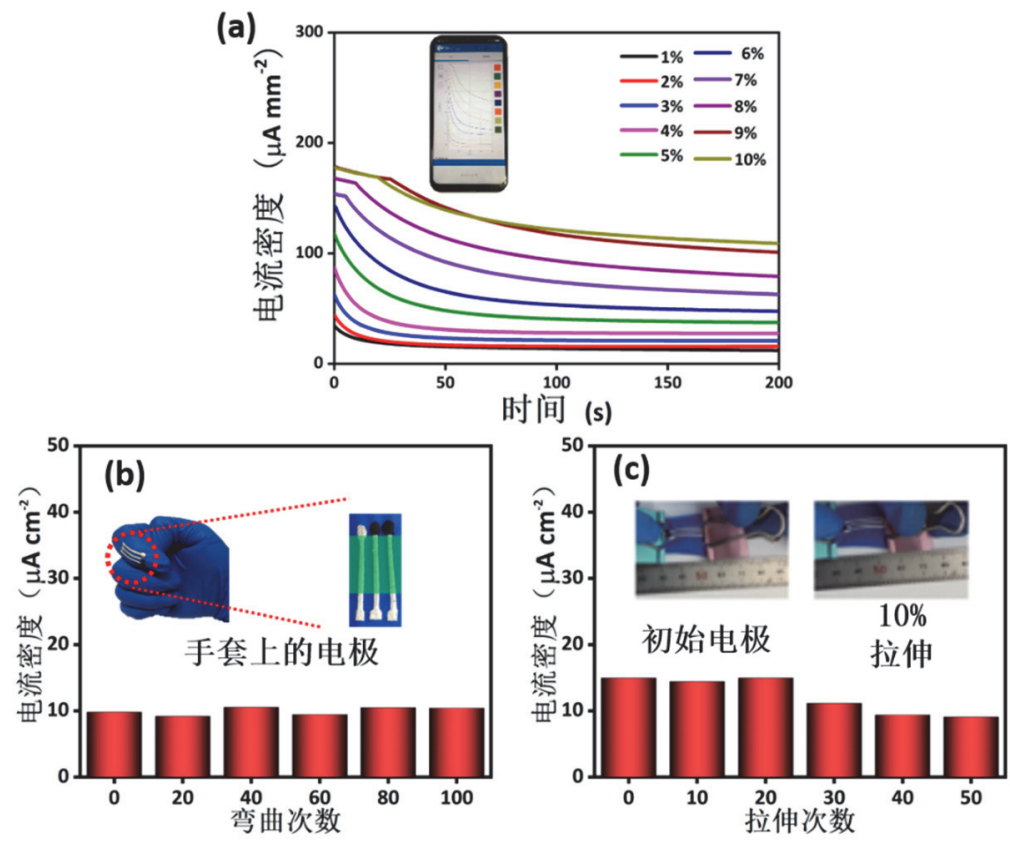

图6 (a) 通过蓝牙装置在手机上测得的甲醇气体环境中的电流-时间曲线; (b) 甲醇传感器在 $5 \% V / V$ 气体环境中的 弯曲性能测试; (c) 甲醇传感器在 $5 \% V / V$ 气体环境中的拉伸性能测试 


\section{3 结语}

本文设计开发了一个综合性创新实验, 即可穿戴智能气液双功能甲醇电化学传感器, 包括还原 氧化石墨烯催化剂的无机合成及结构表征、还原氧化石墨烯修饰电极的电化学性能研究及甲醇检测 应用等相关内容。本实验将电化学分析方法的原理、操作和应用知识充分融合, 可以培养学生综合 运用电化学知识解决问题的能力; 综合了无机化学、仪器分析、材料化学等相关领域的知识内 容, 是一个具有一定难度与挑战性的综合性化学实验。该实验可操作性强、内容具有前沿性, 有利 于培养学生的学习能力和实践能力, 是一个非常值得推荐的综合实验项目。本实验安排为 6 学时, 其 中还原氧化石墨烯的制备为 4 学时、丝网印刷电极的制备为 1 学时、电化学性能测试与甲醇检测为 1 学 时, 如需进一步拓展实验内容, 可结合仪器分析实验对催化剂表征及性能分析为2学时。

通过开展本实验, 可以促使学生复习和串联以往知识点, 了解和掌握相关领域知识内容, 做到 对知识的融会贯通; 可以帮助学生深入了解科学研究热点, 提升对学习科学知识的热爱和学以致用 的领悟能力, 为本科毕业设计或者将来走上科研之路形成良好的知识储备和心理准备; 可以提高学 生的安全防范意识, 并能利用所学化学专业知识解决环境污染问题等社会实际需求, 为人类谋福 利、做贡献。

\section{参 考 文 献}

[1] 间春霞. 创新创业理论研究与实践, 2021, No. 15, 181.

[2] 才金玲, 唐娜, 崔永岩. 黑龙江教育(高教研究与评估), 2021, No. 6, 1 .

[3] 王奇峰, 项金宝. 大学化学, 2022, 37 (2), 2106042.

[4] 何术. 化学工程与装备, 2013, No. 12, 198.

[5] 董绍俊, 车广礼, 谢远武. 化学修饰电极. 北京: 科学出版社, 2003: 3 .

[6] 王钦卓, 白雷, 钱晓否, 李智敏, 万广平, 宋寒. 传感器与微系统, 2020, No. 11, 1 .

[7] Yu, L.; Zhang, L.; Song, H.; Jiang, X.; Lv, Y. CrystEngComm 2014, 16 (16), 3331.

[8] Gong, W.; Hou, C.; Zhou, J.; Guo, Y.; Zhang, W.; Li, Y.; Zhang, Q.; Wang, H. Nat. Commun.2019, 10 (1), 868.

[9] 胡明江, 崔秋娜, 虞婷婷, 吕春旺. 分析化学, 2018, No. 46,1201.

[10] Choi, S. M.; Seo, M. H.; Kim, H. J.; Kim, W. B. Carbon 2011, 49 (3), 904.

[11] Gao, Z.; Li, M.; Wang, J.; Zhu, J.; Zhao, X.; Huang, H.; Zhang, J.; Wu, Y.; Fu, Y.; Wang, X. Carbon 2018, 139, 369.

[12] Wang, Y.; Wang, S.; Li, F.; Wang, Y.; Zhang, H.; Sun, C. ACS Omega 2018, 3 (12), 16850.

[13] Yang, Z.; Li, M.; Cui, P.; Zhang, G.; Jiang, X.; Wang, Y. ACS Sustainable Chem. Eng. 2018, 6 (11), 14026.

[14] 李露, 曹欢玲. 广东化工, 2021, 48 (15), 325.

[15] Qian, W.; Hao, R.; Zhou, J.; Eastman, M.; Manhat, B. A.; Sun, Q.; Goforth, A. M.; Jiao, J. Carbon 2013, 52, 595.

[16] Narayanamoorthy, B.; Datta, K. K. R.; Eswaramoorthy, M.; Balaji, S. ACS Catal. 2014, 4 (10), 3621.

[17] Zhu, N.; Han, S.; Gan, S.; Ulstrup, J.; Chi, Q. Adv. Funct. Mater. 2013, 23 (42), 5297.

[18] Xiong, B.; Zhou, Y.; Zhao, Y.; Wang, J.; Chen, X.; O’Hayre, R.; Shao, Z. Carbon 2013, 52, 181. 